\title{
Management of depression in adults
}

\author{
Markku Timonen,, ${ }^{1}$ Timo Liukkonen²
}

${ }^{1}$ Institute of Health Sciences, University of Oulu, Box 5000, FIN-90014, Finland

${ }^{2}$ Department of Psychiatry, Savonlinna Central Hospital,

Finland

Correspondence to: $\mathrm{M}$ Timonen markku.timonen@oulu.fi

BMJ 2008;336:435-9 doi:10.1136/bmj.39478.609097.BE
A study by the World Health Organization ranked depression the fourth global burden of disease and found it to be the largest non-fatal burden of disease, with nearly $12 \%$ of total years lived with disability. ${ }^{1}$ According to the cross sectional WHO world health survey, carried out in all regions of the world $(60$ countries), the one year prevalence of a depressive episode (international classification of diseases, 10th revision) was $3.2 \%$ (95\% confidence interval $3.0 \%$ to $3.5 \%)$. In patients with several medical conditions the prevalence of depression exceeds that of the general population, ${ }^{2}$ with $5-10 \%$ of patients affected in primary care and $10-14 \%$ of patients under general hospital care. ${ }^{3}$ The diagnosis and treatment of depression by general practitioners is not, however, always optimal. ${ }^{45}$ We review the presentation and assessment of depression and discuss the options for its effective treatment and management.

\section{Why is depression so difficult to diagnose? \\ According to cross sectional studies $50-70 \%$ of patients with depression in primary care remain undetected, with somatisation being one of the most important single problems associated with a missed diagnosis. ${ }^{5}$ Given that about two thirds of depressed patients present mainly with somatic symptoms, ${ }^{6}$ detecting depression in connection with somatisation should be a core professional skill of doctors. During consultation the discussion should move away from somatic symptoms to emotional health by asking patients open questions on what they think is the cause of their physical symptoms. It is also worth inquiring about possible life events preceding the symptoms. \\ The detection of depression can be improved by training in mental health and screening. ${ }^{5}$ Patients at high risk in both primary care and general hospital settings (for example, those with chronic medical}

\section{Sources and selection criteria}

We searched PubMed, the Cochrane database of systematic reviews, and citation lists of relevant publications using the subject headings and key words "depression", "major depression", "treatment", "management", "psychosocial", and "pharmacological." We also searched guidelines from the National Institute for Health and Clinical Excellence, the American Psychiatric Association, and the World Federation of Societies of Biological Psychiatry. illness, chronic pain syndromes, recent life events, fair or poor self rated health, and unexplained physical symptoms $\mathrm{s}^{7}$ ) can be screened for depression by asking two questions on mood and interest (box 1). ${ }^{8}$ Eventually, however, the diagnosis is a clinical one, which must be obtained through consultation to determine whether the criteria for depression are met (fig 1). Although drugs and general medical conditions such as hypothyroidism, hyperthyroidism, Huntington's disease, Cushing's disease, and Addison's disease do not represent a substantial public health problem as a causal factor for depression, management, when appropriate, must be directed at the underlying condition rather than the depressive symptoms. More commonly several physical illnesses occur with depression; if so, treatment must be directed at the depression as well as the illness. ${ }^{7}$ Depression also occurs commonly with anxiety disorders. ${ }^{5}$ Depression requires treatment first when it is considered the primary diagnosis.

\section{What should be taken into account while building a management plan?}

Guidelines for depression emphasise the importance of an effective doctor-patient relationship while an appropriate and comprehensive management plan is being negotiated (box 2), and this relationship should continue throughout treatment. Factors that also need to be taken into account when designing the management plan and deciding on a treatment setting are patients' preferences, concomitant psychiatric and physical disorders, concurrent drugs, patients' experiences with previous treatments, the severity of depressive symptoms or subtypes of depression, risk of suicide, and availability of treatment options. ${ }^{9-11}$

As single component interventions, such as screening alone, have been shown to be ineffective in the management of depression in primary care, complex multifaceted educational and organisational interventions have been developed to improve outcomes. ${ }^{45} \mathrm{~A}$ recent systematic review and metaregression found that the effective determinants of "collaborative care" (one particular multifaceted intervention), were systematic identification of patients, case managers with a professional background, and regular supervision of case managers by specialists. ${ }^{12}$ 
Despite a lack of corresponding studies outside the United States ${ }^{11}{ }^{12}$ the collaborative care model seems promising for enhancing treatment outcomes and bridging the gap between primary and secondary care, given that it allows patients to be managed by case managers or primary care providers under specialist supervision without the need for treatment elsewhere.

\section{Which treatment setting?}

More than $80 \%$ of patients with depression are managed and treated in primary care. ${ }^{11}$ The treatment of depressed patients in secondary care should be skewed towards those with more severe disease. Specialist treatment is indicated for patients displaying psychotic features or active suicidality. Moreover, specialists should be involved when patients are resistant to treatment or have recurrent depression. A stepped care model (fig 2), originally introduced by the National Institute for Health and Clinical Excellence (NICE), lists the needs associated with treating or managing depression at different service levels. ${ }^{1011}$

\section{How is depression managed in the acute phase?}

Although most of the interventions for treating depression have been carried out in high income

\section{International classification of disease, 10th revision \\ Depressive episode \\ Typical symptoms: \\ - Depressed mood \\ - Loss of interest and enjoyment \\ - Increased fatiguability \\ Common symptoms: \\ - Reduced concentration and attention \\ - Reduced self esteem and self confidence \\ - Ideas of guilt and unworthiness \\ - Bleak and pessimistic views of future \\ - Ideas or acts of self harm or suicide \\ - Disturbed sleep \\ - Diminished appetite \\ F32.0 Mild depressive episode - at least two o the typical symptoms and at least two of the common symptoms, and none should present to an intense degree}

F32.1 Moderate depressive episode - at least two of the typical symptoms and at least three of the common symptoms

F32.2 Severe depressive episode - all the typical symptoms and at least four of the common symptoms, and some should be of severe intensity

F32.3 Severe depressive episode with psychotic symptoms - criteria given for F32.2 and in which delusions, hallucinations, or depressive stupors are present

Minimum duration of an episode is about two weeks, and patient has never had a manic or hypomanic episode

Further depressive episodes should be classified under one of the subdivisions of recurrent depressive disorder (F33.1-3)
Diagnostic and Statistical Manual of Mental Disorders, fourth edition

\section{Major depressive episode}

A Five (or more) of the following symptoms have been present during the same two week period and at least one of the symptoms is either depressed mood or loss of interest or pleasure. Each symptom should be present most of the day or nearly every day:

- Depressed mood

- Diminished interest or pleasure in almost all activities

- Significant weight loss or weight gain or decreased or increased appetite

- Insomnia or hypersomnia

- Psychomotor agitation or retardation

- Fatigue or loss of energy

- Feeling of worthlessness or inappropriate guilt

- Diminished ability to think or concentrate or indecisiveness

- Recurrent thoughts of death, suicidal ideation, suicide attempt, or suicide plan

B The symptoms do not meet criteria for a mixed episode

C The symptoms cause clinically significant distress or impairment in social, occupational, or other important areas of functioning

D The symptoms are not due to the direct physiological effects of a substance (for example, a drug of misuse, a medication) or a general medical condition (for example, hypothyroidism)

E The symptoms are not better accounted for by bereavement

296.2x Major depressive disorder, single episode

- Presence of a single major depressive episode

- The major depressive episode is not better accounted for by schizoaffective and is not superimposed on schizophrenia or schizophereform, delusional or psychotic disorder otherwise specified

- A manic, a mixed, or a hypomanic episode has never occurred

Presence of two or more major depressive episodes justifies a diagnosis of major depressive disorder, recurrent $296.3 \mathrm{x}$

\section{Box 1: Two screening questions for depression}

During the past month have you often been bothered by feeling down, depressed, or hopeless?

During the past month have you often been bothered by having little interest or pleasure in doing things?

If patients answer yes to either question then the specificity of screening can be further increased by asking them whether they want help with their problems

countries, an increasing amount of evidence comes from low income and middle income countries. ${ }^{13}$ Whatever the treatment for depression the primary goal in the acute phase is remission of symptoms, ${ }^{910}$ given that remission is associated with a better prognosis than the improvement of depressive symptoms without remission. ${ }^{14}$

\section{Psychological treatments}

In mild depression, based mostly on clinical experience, NICE recommends a further assessment within two weeks (watchful waiting) for those not agreeing to intervention and for those who healthcare professionals believe may recover spontaneously without intervention. ${ }^{11}$ Otherwise the recommended first line treatments for mild depression on the basis of substantial research based evidence are guided self help as well as brief psychological interventions (6-8 sessions) including problem solving therapy, brief cognitive behaviour therapy, and counselling. ${ }^{11}$ Evidence also shows that problem solving therapy, brief cognitive behaviour therapy, and counselling are effective in moderate depression. ${ }^{11}$ On the basis of substantial evidence from a randomised controlled trial (274 participants), a NICE guideline recommends one of three types of computerised cognitive behaviour therapy as optional for the treatment of mild and moderate depression. ${ }^{15}$ Antidepressants are notrecommended for the initial treatment of mild depression because of minimal evidence to support their efficacy and a poor risk-benefit ratio. ${ }^{1011}$

Several structured psychological interventions (1620 sessions) were found to be effective for moderate to severe depression. Firstly, research based evidence favouring individual cognitive behaviour therapy over a wait list control is good. ${ }^{11}$ Secondly, in a small number of well designed randomised controlled trials interpersonal therapy was more effective than placebo or usual care by a general practitioner. ${ }^{11}$ Group interpersonal therapy was also found to be efficacious in a randomised controlled trial in a low income country. ${ }^{16}$ Finally, data from two small randomised controlled trials to treat depression suggest that marital therapy (treatment for couples with depressed spouses, when relationship problems are associated with depression) is more efficacious than no therapy, ${ }^{17}$ but clear evidence is lacking for the efficacy of family therapy and short term psychodynamic psychotherapy. ${ }^{911} 18$ Although patients generally prefer these psychological treatments, they are not always available. ${ }^{19}$ 
Box 2: Components of a comprehensive management plan for depression. Adapted from American Psychiatric Association and World Federation of Societies of Biological Psychiatry guidelines $^{910}$

- Determine the pharmacological or psychosocial treatment

- Determine the treatment setting

- Establish and maintain a therapeutic alliance

- Monitor and reassess the patient's psychiatric status in the course of treatment

- Monitor the patient's response to treatment

- Reassess the adequacy of diagnosis when appropriate

- Monitor possible side effects and physical condition

- Enhance treatment adherence

- Educate patients and their families about the nature of the illness (psychoeducation)

\section{Pharmacological treatment}

Robust evidence from numerous randomised controlled trials shows that tricyclic antidepressants, selective serotonin reuptake inhibitors (SSRIs), and selective serotonin and norepinephrine reuptake inhibitors are more efficacious than placebo for treating major depressive disorder. ${ }^{10}$ Most of the investigations were, however, sponsored by the pharmaceutical industry. ${ }^{1120}$ The evidence for serotonergic-noradrenergic mirtazapine is also well documented. ${ }^{1011}$

A Cochrane review and meta-analyses of 194 randomised controlled trials found that the tricyclic antidepressant amitriptyline (one of the reference compounds for pharmacological treatment of depression) can still be regarded at least as efficacious as other tricyclic antidepressants or SSRIs, although it is not as well tolerated as SSRIs. ${ }^{21}$ Also, according to the few randomised controlled trials carried out in low income and middle income countries, ${ }^{13}$ a multifaceted stepped care model including treatment with amitriptyline, imipramine, or fluoxetine for those with severe or persistent depression by structured pharmacotherapy programme, ${ }^{22}$ and fluoxetine alone ${ }^{23}$ have been shown to be efficacious in treating depression. Generally,

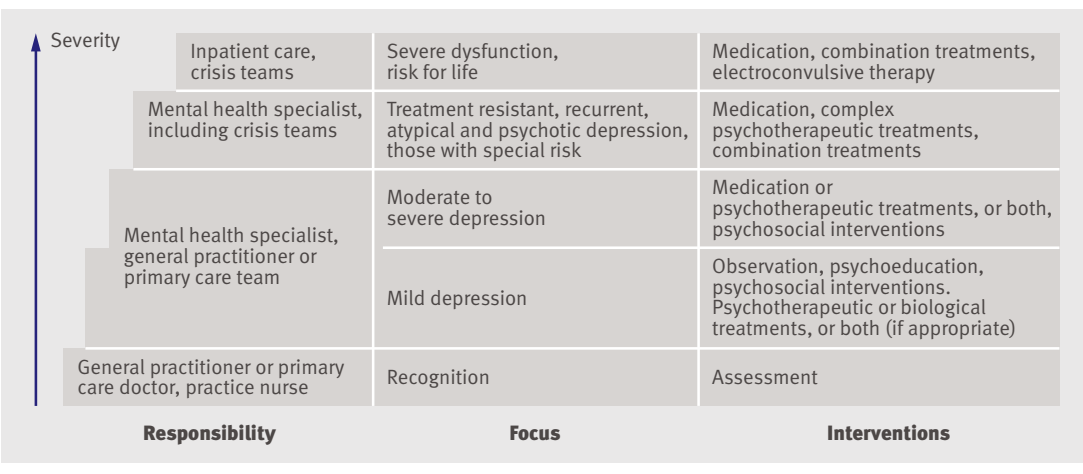

$\overline{\text { Fig } 2}$ | Stepped care model for treatment and management of depression in primary care. Adapted from World Federation of Societies of Biological Psychiatry guideline, original idea from NICE guideline $^{1011}$ strong evidence suggests that SSRIs are better tolerated than tricyclic antidepressants. ${ }^{21}$ NICE recommends SSRIs as first choice drugs for depression. ${ }^{11}$

A recent systematic review and meta-analysis of 93 published and unpublished randomised controlled trials showed that serotonergic-noradrenergic antidepressant drugs had a modest efficacy advantage over SSRIs, the number needed to treat to benefit being about $24 .{ }^{24}$ Irreversible monoamine oxidase inhibitors, an older class of antidepressants, are not considered first line treatments because of their potentially fatal side effects. ${ }^{10} \mathrm{We}$ believe that moclobemide (a reversible monoamine oxidase inhibitor) is not suitable as first line treatment for depression owing to the potential risk of serotonergic syndrome and the unconditional need for a wash-out period while switching antidepressants.

All guidelines ${ }^{9-11}$ emphasise the importance of clinical judgment when applying the evidence from clinical trials to individual patients. For example, participants in clinical trials are often free of common concomitant psychiatric or physical conditions, and thus the generalisability of the results to real world circumstances are questionable. When prescribing antidepressants, potential side effects and interactions with current drugs need to be considered along with a patients' preference and experience of previous treatments. Furthermore, antidepressants are associated with poor rates of remission-in one trial remission occurred in only $36.8 \%$ of participants treated with an SSRI. ${ }^{14}$ Finally, the better treatment outcomes with multifaceted interventions rather than with treatments as usual ${ }^{4}$ also highlight the importance of factors other than antidepressants in the management of depression.

\section{Combination of pharmacological and psychological treatments}

Strong evidence shows efficacy of combined antidepressant and cognitive behaviour therapy over antidepressant alone in moderate to severe depression and in chronic depression. ${ }^{11}$ One relatively small

Box 3: Evaluating the efficacy of treatment for depression. Adapted from World Federation of Societies of Biological Psychiatry guideline ${ }^{10}$

- Non-response-25\% or less decrease in symptom severity compared with baseline

- Partial response-26-49\% decrease in symptom severity compared with baseline

- Response- $50 \%$ or greater decrease in severity of symptoms compared with baseline

- Remission-absence of symptoms defined by absolute scale (for example, score of $\leq 7$ on Hamilton rating scale for depression)

Several patient self rating and observer rating scales are available-for example, Beck depression inventory, 16 item clinician rated quick inventory of depressive symptomatology, Hamilton rating scale for depression, Montgomery-Asberg depression rating scale 


Box 4: Factors associated
with increased risk for
depression recurrence.
Adapted from World
Federation of Societies of
Biological Psychiatry
guideline ${ }^{10}$
- Three or more episodes
of major depression
- High prior frequency of
recurrence
- Previous episode in
past year
- Residual symptoms
during continuation
phase treatment
- Severity of episodes
(for example,
suicidality and
psychotic features)
- Long previous
episodes
- Relapse after drug
withdrawal

randomised controlled trial found interpersonal therapy combined with antidepressants to be more efficacious than interpersonal therapy alone. ${ }^{11}$

\section{How should treatment efficacy be evaluated?}

Response to treatment must be carefully monitored irrespective of the chosen treatment modality, and structured measures of symptom severity and functional status can be used. ${ }^{9}$ According to the World Federation of Societies of Biological Psychiatry guideline for primary care, the efficacy of antidepressant treatment should be evaluated 2-4 weeks after the primary care provider starts treatment, using patient self rating or observer rating scales (box 3). ${ }^{10}$ This evaluation can also be done by other healthcare professionals in the primary care team. ${ }^{414}$ It should be remembered, however, that remission is a clinical judgment that cannot be replaced by rating scales.

\section{What should be done if patients do not respond to treatment in the acute phase?}

If the response to antidepressants is insufficient after 2-4 weeks then strategies to optimise treatment should be implemented. ${ }^{10}$ Normal clinical practice at this point is to increase the antidepressant dose to the upper level of the standard dose. ${ }^{1011}$ On the basis of a systematic review of eight randomised controlled trials on dose escalation, evidence is insufficient for dose escalation of SSRIs. ${ }^{25}$

If patients show no improvement after four weeks of pharmacological treatment ${ }^{10}$ or at least moderate improvement after 4-8 weeks of psychological ${ }^{9}$ treatment then the diagnosis and compliance to treatment should be reassessed. If the diagnosis is still depression, a new management plan must be negotiated, taking into account patients' preferences and availability of treatments: options to consider when psychological treatment has been part of the management plan are changing intensity or type of psychotherapy, changing to pharmacological treatment, and combining antidepressant with ongoing psychotherapy. ${ }^{9}$ The general

\section{Ongoing research}

To identify the active elements of the multifaceted interventions in the management of depression

In the Cochrane Collaboration, as part of the multiple meta-analyses of new generation antidepressants (MANGA) study, separate reviews are comparing the efficacy of commonly used new compounds with other antidepressants

New antidepressants with novel mechanisms of action, such as melatonergic agomelatine, are being developed

Ongoing research is investigating technological adaptations on psychotherapies, especially computerised cognitive behaviour psychotherapy

A promising treatment for depression, transcranial magnetic stimulation, might have significant antidepressant effects, but larger multicentre studies are needed to assess this modality

Therapeutic brain stimulation through delivery of pulsed electrical impulses to the left cervical vagus nerve, vagus nerve stimulation, is a novel approach for treatment resistant depression

\section{Tips for non-specialists}

A definitive diagnosis of depression should be assessed within one or two weeks of the initial visit

Rule out bipolar disorder by questioning whether mania or hypomania has occurred earlier in life

In addition to suicidal ideas and intent ask patients directly about hallucinations and delusions

Become familiar with the efficacy of some locally available first line and second line antidepressants and their short and long term side effects and interactions with other drugs

A member of the primary care team should learn one of the observer rating scales for depression to evaluate the efficacy of treatment

Patients should be seen weekly or biweekly during the acute phase and every month or bimonthly during the continuation phase

consensus on antidepressants is to switch to another one. A systematic review of eight randomised controlled trials and 23 open studies found that any switch within or between classes of antidepressants seems legitimate after the first use of an SSRI. ${ }^{26}$

\section{How is depression treated in a continuation phase?}

The goal of the continuation phase is to stabilise the remission and to prevent relapse. ${ }^{10}$ Antidepressants should be continued for about six months after remission because strong evidence shows that this reduces the risk of relapse. ${ }^{11}$ The antidepressant and dose successfully used in the acute phase should be given during the continuation phase. ${ }^{10}$ If no relapse occurs and the patient does not need prophylactic treatment, then gradual discontinuation of the antidepressant over four weeks is recommended at the end of this phase, ${ }^{9-11}$ with the patient being informed about possible discontinuation or withdrawal symptoms.

Studies of psychotherapies in continuation and maintenance phases are largely lacking. ${ }^{9}$ A recent systematic review and meta-analysis of four clinical trials (three randomised controlled trials) found that cognitive behaviour therapy during the continuation phase significantly reduced the rate of relapse and recurrence compared with controls who received assessment only. The efficacy of the therapy, based on five small randomised controlled trials, was similar to that of other active treatments such as pharmacotherapy. ${ }^{27}$

\section{Which patients need maintenance or prophylactic treatment?}

Given that depression has a strong tendency for recurrence $(30-40 \%$ of patients with depression in primary care $\left.{ }^{28}\right)$, prophylactic therapy is indicated for patients at high risk of recurrence (box 4). ${ }^{10} \mathrm{~A}$ consultant psychiatrist or specialist in secondary care should evaluate the need for maintenance or prophylactic treatment. ${ }^{1011}$ 


\section{Additional educational resources}

National Institute for Health and Clinical Excellence, Depression (http://guidance.nice.org uk/CG23)

Clinical guideline, based on the best available evidence, on the appropriate treatment within the National Health Service in England and Wales

Royal College of Psychiatry (www.rcpsych.ac.uk)

Structured leaflets on depression and related issues in different languages for patient education purposes

Cochrane Database of Systematic Reviews (www3.interscience.wiley.com/cgi-bin/ mrwhome/106568753/HOME)

High quality, independent, systematic reviews on psychotherapies and pharmacotherapies Information for patients

National Institute for Health and Clinical Excellence. Depression: information for the public (amended) (http://guidance.nice.org.uk/CG23/publicinfo/pdf/English)

Freely available information on the care people with depression can expect to receive from their general practitioner or other healthcare professionals

Royal College of Psychiatry (www.rcpsych.ac.uk)

Freely available information for the public on depression, in different languages

We thank V Benno Meyer-Rochow for checking the English language of this paper.

Contributors: Both authors researched and drafted the sections with which they were most familiar. MT combined the separate sections. Both authors contributed to and approved all versions of the manuscript. MT is the guarantor.

Competing interests: MT has been reimbursed by GlaxoSmithKline, Eli

Lilly, Novartis, and $\mathrm{H}$ Lundbeck for attending six conferences, was paid by Eli Lilly and Astra Zeneca for speaking on seven different occasions, and has served as coauthor or principal investigator in international multicentred placebo controlled clinical trials organised and financed by five different medical companies. TL has been reimbursed by $\mathrm{H}$ Lundbeck, Astra Zeneca, and Wyeth for attending three conferences, has been paid for speaking on two occasions by Pfizer, and has received personal funds for research from $\mathrm{H}$ Lundbeck.

Provenance and peer review: Commissioned and externally peer reviewed.

1 Ustün TB, Ayuso-Mateos JL, Chatterji S, Mathers C, Murray CJ. Global burden of depressive disorders in the year 2000. Br J Psychiatry 2004;184:386-92.

2 Moussavi S, Chatterij S, Verdes E, Tandon A, Patel V, Ustun B. Depression, chronic diseases, and decrements in health: results from the world health surveys. Lancet 2007;370:851-8.

3 Katon WJ. Clinical and health services relationships between major depression, depressive symptoms, and general medical illness. Biol Psychiatry 2003;54:216-26.

4 Williams JW Jr, Gerrity M, Holsinger T, Dobscha S, Gaynes B, Dietrich A. Systematic review of multifaceted interventions to improve depression care. Gen Hosp Psychiatry 2007;29:91-116.

\section{SUMMARY POINTS}

Somatisation is one of the most important single problems associated with a missed diagnosis of depression in primary care

Depression should be managed comprehensively and the efficacy of treatment evaluated for the first time within one month

When response to treatment is poor the diagnosis and compliance with treatment should be reassessed

\section{Psychosocial treatments are first line treatments for mild depression}

Structured psychological interventions, such as cognitive behaviour therapy and interpersonal therapy, and antidepressants are effective in moderate to severe depression

Evidence suggests that combined antidepressant and cognitive behaviour therapy is more efficacious than antidepressants alone in moderate to severe depression and chronic depression
5 Tylee A, Walters P. Underrecognition of anxiety and mood disorders in primary care: why does the problem exist and what can be done? JClin Psychiatry 2007;68(suppl 2):27-30.

6 Tylee A, Gandhi P. The importance of somatic symptoms in depression in primary care. Prim Care Companion J Clin Psychiatry 2005;7:167-76.

7 Williams JW Jr, Noël PH, Cordes JA, Ramirez G, Pignone M. Is this patient clinically depressed? JAMA 2002;287:1160-70.

8 Arroll B, Goodyear-Smith F, Kerse N, Fishman T, Gunn J. Effect of the addition of a "help" question to two screening questions on specificity for diagnosis of depression in general practice: diagnostic validity study. BMJ 2005;331:884-6.

9 American Psychiatric Association. Practice guideline for the treatment of patients with major depressive disorder (revision). Am J Psychiatry 2000;157(suppl 4):1-45.

10 Bauer M, Bschor T, Pfennig A, Whybrow PC, Angst J, Versiani M, et al. World Federation of Societies of Biological Psychiatry (WFSBP) guidelines for biological treatment of unipolar depressive disorders in primary care. World J Biol Psychiatry 2007;8:67-104.

11 National Institute for Clinical Excellence. Depression: management of depression in primary and secondary care. London: NICE, 2004. (National Clinical Practice Guideline No 23.)

12 Bower P, Gilbody S, Richards D, Fletcher J, Sutton A. Collaborative care for depression in primary care. Making sense of a complex intervention: systematic review and meta-regression. Br J Psychiatry 2006;189:484-93.

13 Patel V, Araya R, Chatterjee S, Chisholm D, Cohen A, De Silva M, et al. Treatment and prevention of mental disorders in low-income and middle-income countries. Lancet 2007;370:991-1005.

14 Rush AJ, Trivedi MH, Wisniewski SR, Nierenberg AA, Stewart JW, Warden D, et al. Acute and longer-term outcomes in depressed outpatients requiring one or several treatment steps: a STAR* D report. Am J Psychiatry 2006;163:1905-17.

15 National Institute for Health and Clinical Excellence. Technological Appraisal 97 Computerised cognitive behaviour therapy for depression and anxiety: Guidance. 22 Feb 2006. http://guidance. nice.org.uk/TA97/guidance/pdf/English.

16 Bolton P, Bass J, Neugebauer R, Verdeli H, Clougherty KF, Wickramaratne $\mathrm{P}$, et al. Group interpersonal psychotherapy for depression in rural Uganda. JAMA 2003;289:3117-24.

17 Barbato A, D’Avanzo B. Marital therapy for depression. Cochrane Database Syst Rev 2006; (Issue 2):CD004188. doi: 10.1002/ 14651858.CD004188.pub2.

18 Henken HT, Huibers MJH, Churchill R, Restifo K, Roelofs J. Family therapy for depression. Cochrane Database Syst Rev 2007; (Issue 3):CD006728. doi: 10.1002/14651858.CD006728.

19 Wolf NJ, Hopko DR. Psychosocial and pharmacological interventions for depressed adults in primary care: a critical review. Clin Psychol Rev 2008;28:131-61.

20 Melander H, Ahlqvist-Rastad J, Meijer G, Beermann B. Evidence biased medicine-selective reporting from studies sponsored by pharmaceutical industry: review of studies in new drug applications. BMJ 2003;326:1171-3.

21 Guaiana G, Barbui C, Hotopf M. Amitriptyline for depression. Cochrane Database Syst Rev 2007; (Issue 3):CD004186. doi: 10.1002/14651858.CD004186.pub2.

22 Araya R, Rojas G, Fritsch R, Gaete J, Rojas M, Simon G, et al. Treating depression in primary care in low-income women in Santiago, Chile: a randomised controlled trial. Lancet 2003;361:995-1000.

23 Patel V, Chisholm D, Rabe-Hesketh S, Dias-Saxena F, Andrew G, Mann A. Efficacy and cost-effectiveness of drug and psychological treatments for common mental disorders in general health care in Goa, India: a randomised, controlled trial. Lancet 2003;361:33-9.

24 Papakostas GI, Thase ME, Fava M, Craig Nelson J, Shelton RC. Are antidepressant drugs that combine serotonergic and noradrenergic mechanisms of action more effective than the selective serotonin reuptake inhibitors in treating major depressive disorder? A metaanalysis of studies of newer agents. Biol Psychiatry 2007;62:1217-27.

25 Ruhé HG, Huyser J, Swinkels JA, Schene AH. Dose escalation for insufficient response to standard-dose selective serotonin reuptake inhibitors in major depressive disorder: systematic review. $\mathrm{Br} J$ Psychiatry 2006;189:309-16.

26 Ruhé HG, Huyser J, Swinkels JA, Schene AH. Switching antidepressants after a first selective serotonin reuptake inhibitor in major depressive disorder: a systematic review. J Clin Psychiatry 2006;67:1836-55.

27 Vittengl JR, Clark LA, Dunn TW, Jarret RB. Reducing relapse and recurrence in unipolar depression: comparative meta-analysis of cognitive-behavioural therapy's effects. J Consult Clin Psychol 2007;3:475-88.

28 Van Weel-Baumgarten EM, Schers HJ, van den Bosch WJ, van den Hoogen HJ, Zitman FG. Long-term follow-up of depression among patients in the community and in family practice settings. A systematic review. J Fam Pract 2000;49:1113-20. 